BULLETIN OF THE

AMERICAN MATHEMATICAL SOCIETY

Volume 81, Number 3, May 1975

\title{
INVARIANTS OF 3-MANIFOLDS
}

\author{
BY SYLVAIN E. CAPPELL AND JULIUS L. SHANESON
}

Communicated by Glen Bredon, October 28, 1974

The $\mu$-invariant $\mu(M)$ of an oriented $Z_{2}$-homology 3-sphere $M$ is defined by Hirzebruch in [8], using Rohlin's Theorem [13], to be the mod 16 reduction of the signature of a framed manifold $W$ with $M=\partial W$. In this paper we give a formula for $\mu(M)$ by studying $M$ as a branched dihedral covering space of $S^{3}$. Hilden [7] and Montesinos [9] have independently shown that every closed orientable 3-manifold is actually a 3-fold (dihedral) covering space of $S^{3}$ branched along a knot. ${ }^{1}$ Also see [1], [6] and [12].

Let $\alpha$ be a smooth or piecewise linear oriented knot $S^{1} \subset S^{3}$. Let $V \subset S^{3}$ with $\partial V=\alpha$ be a Seifert surface for $\alpha$. The Seifert form $L=L_{V}$ is the bilinear form of linking numbers of circles in $V$, with respect to a fixed orientation of $S^{3}$, and $L^{\prime}$ is given as $L^{\prime}(x, y)=L(y, x)$. Let $p$ be an odd integer. A knot $\beta$ will be called a $\bmod p$ characteristic knot (in $V$ ) of $\alpha$ if there is an embedding of $S^{1}$ in $V$, with nontrivial homology class $[\beta] \in H_{1}(V)$, so that the composite $S^{1} \subset V \subset S^{3}$ is $\beta$; and if $L(x, \beta)+L(\beta, x) \equiv 0(\bmod p)$ for all $x$ in $H_{1}(V)$.

$A \bmod p$ characteristic $\operatorname{knot} \beta$ for $\alpha$ determines a homomorphism $\rho$ of $\pi_{1}\left(S^{3}-\alpha\right)$ onto the dihedral group $Z_{2} \times{ }_{\omega} Z_{p}$ of order $2 p$. The map $\rho$ is characterized by the requirements that its composition with $Z_{2} \times{ }_{\omega} Z_{p} \rightarrow Z_{2}$ be nontrivial and that, for $x$ in the image of $\pi_{1} V, \rho(x) \in Z_{p} \subset Z_{2} \times_{\omega} Z_{p}$ is the $\bmod p$ reduction of $L(x, \beta)$. Hence $\beta$ determines a $p$-fold dihedral branched covering $M_{\alpha, \beta}$ of $S^{3}$, branched along $\alpha$. It can be shown that every dihedral representation for $\alpha$ and associated branched cover of $S^{3}$ are determined by a characteristic knot for $\alpha$ in $V$. Further, dihedral representations can easily be classified in terms of equivalence classes of characteristic knots. By abuse of notation, we write $M_{\alpha}$ for $M_{\alpha, \beta}$; as "most" knots have at most one (up to conjugacy) dihedral representation of order $2 p$, this notation is usually strictly justified.

AMS(MOS) subject classifications (1970). Primary 55A99, 55A10, 55A25, 55 A40; Secondary 57D65.

1 In fact one can go directly from a Heegard splitting to a description of any orientable 3-manifold as a dihedral branched covering space. 
Let $\alpha_{0}, \cdots, \alpha_{(p-1) / 2}$ be the disjoint oriented circles in $M_{\alpha}$ that lie over $\alpha$, with $\alpha_{0}$ of branching index 1 and $\alpha_{i}$ of index $2,1 \leqslant i \leqslant(p-1) / 2$. Orient $M_{\alpha}$ so that the covering projection has positive degree, and let $v_{i j}$ denote the linking number of $\alpha_{i}$ with $\alpha_{j}, i \neq j$. If $M_{\alpha}$ is a homology sphere, then $v_{i j}$ is an integer, but for a $Z_{2}$-homology sphere $v_{i j}$ will in general be a fraction with odd denominator. Let

$$
v_{i i}=-\left(\sum_{j=1 ; j \neq i}^{(p-1) / 2} v_{i j}+v_{i 0} / 2\right), \quad v_{0}=-2 \sum_{i=1}^{(p-1)^{/ 2}} v_{0 i} .
$$

Let $J$ be the matrix $\left(\left(v_{i j}\right)\right)_{1<i, j \leqslant(p-1) / 2}$. K. Perko introduced $v_{0}$ and has computed $v_{i j}$ and $v_{0}$ for many knots.

Let $\Sigma_{\beta}$ be the $p$-fold cyclic branched cover of $S^{3}$, branched along $\beta$, and oriented so that the covering projection $\tau$ has positive degree. For $p$ a prime-power $\Sigma_{\beta}$ is a rational homology sphere [5]. ${ }^{2}$ Let $T$ be a covering translation corresponding to a meridian about $\beta$. Then

$$
\tau^{-1} V=\bar{V} \cup_{\beta} T(\bar{V}) \cup_{\beta} \cdots \cup_{\bar{\beta}} T^{p-1}(\bar{V})
$$

where $\tau \mid \bar{V}: \bar{V} \rightarrow V$ is a homeomorphism, and where $\bar{\beta}=\tau^{-1}(\beta)$. Let $z_{1}$, $\cdots, z_{r}$ be elements in the image of $H_{1}(V-\beta)$ in $H_{1}(V)$ which, together with [ $\beta$ ], form a basis (over $Q$ ) for this image. Let $A_{i}$ be the matrix whose $(j, k)$ th entry is the linking number in $\Sigma_{\beta}$ of $(\tau \mid \bar{V})_{*}^{-1} z_{j}$ and $T_{*}^{i}\left((\tau \mid \bar{V})_{*}^{-1} z_{k}\right), 1 \leqslant i \leqslant$ $p-1$. Let $A$ have the $(j, k)$ th entry $L_{V}\left(z_{j}, z_{k}\right)$. Let $R=\left(\left(R_{i j}\right)\right), 1 \leqslant i, j \leqslant$ $(p-1) / 2$ be the matrix of blocks where, with subscripts modulo $p$,

$$
R_{i j}=A_{i-j}+A_{j-i}-A_{i+j}-A_{-i-j}, \quad i \neq j,
$$

and

$$
R_{i i}=A+A^{\prime}-2\left(A_{1}+\cdots+A_{p-1}\right)-A_{2 i}-A_{-2 i} .
$$

For any knot $\eta$ in a $Z_{2}$-homology sphere, let $\Delta_{\eta}(t)$ denote its Alexander polynomial.

Let $\hat{\alpha}$ be any knot obtained from $\alpha_{1}, \cdots, \alpha_{(p-1) / 2}$ by connected sum using $(p-3) / 2$ paths joining them. (Such paths may be described by lifting suitable paths from $\alpha$ to itself in $S^{3}$.)

For any fraction $p / q, p$ and $q$ odd, let $\varphi(p / q)=0$ if $p / q \equiv \pm 1(\bmod 8)$ and $\varphi(p / q)=8$ if $p / q \equiv \pm 3(\bmod 8)$.

${ }^{2}$ When $p$ is not a prime-power and $\Sigma_{\beta}$ not a rational homology sphere, the definition of the matrix $R$ is slightly more complicated. 
If $N$ is a Hermitian matrix, let $\sigma(N)$ denote its signature. Let $\psi$ be a primative $p$ th root of unity. Let $B$ be a Seifert matrix for $\beta$.

THEOREM. Suppose that the branched $p$-fold dihedral covering space $M_{\alpha}$ of $S^{3}$ is a $Z_{2}$-homology sphere. Assume that $v_{i, 0} \equiv 2$ (4) for $1 \leqslant i \leqslant$ $(p-1) / 2$. Then the following holds modulo 16 :

$$
\begin{aligned}
\mu\left(M_{\alpha}\right)= & \sum_{i=1}^{p-1} \sigma\left(B+B^{\prime}-B \psi^{i}-B^{\prime} \psi^{-i}\right)+\left(\frac{p-1}{2}\right) \varphi\left(\Delta_{\alpha}(-1)\right) \\
& +\varphi(p) L_{V}([\beta],[\beta])+\varphi\left(\Delta_{\hat{\alpha}}(-1) / \Delta_{\hat{\alpha}}(1)\right)-\left(v_{0} / 4\right)+\sigma(J)-\sigma(R) .
\end{aligned}
$$

The terms on the right of this formula are readily calculable. Note that some of the terms vary with the choice of characteristic knot $\beta$. (However, for suitable dihedral covers of ribbon knots these terms contribute zero. This gives a simple obstruction to a knot being a ribbon knot.) For a homology sphere $M_{\alpha}$, the terms on the right are integers. For "bushel baskets" [6] of knots $\alpha, \pi_{1}\left(M_{\alpha}\right)=0 .^{3}$

some of the terms vary with the choice of characteristic knot $\beta$. For a homology sphere $M_{\alpha}$, the terms on the right are integers. For "bushel baskets" [6] of knots $\alpha, \pi_{1}\left(M_{\alpha}\right)=0 .^{3}$

The condition on $v_{i, 0}$ seems to be satisfied in all known cases for $M_{\alpha}$ a $Z_{2}$-homology sphere [10], [11]. It implies that $\operatorname{det} J \neq 0$, which suffices for the theorem. Therefore it seems reasonable to conjecture at least that $\operatorname{det} J \neq$ 0 if $M_{\alpha}$ is a $Z_{2}$-homology sphere. For $p=3$, we can show that $v_{1,0}=2$ ( $\bmod 4)$. (See [2] and [10] for 2-bridge knots.) Hence the Theorem applies in this case. By [7] and [9] the case $p=3$ of our formula applies to every $Z_{2}$ homology sphere.

\section{REFERENCES}

1. J. W. Alexander, Note on Riemann spaces, Bull. Amer. Math. Soc. 26 (1919), $370-372$.

2. C. Bankwitz and H. G. Schumann, Über Viergeflechte, Abh. Math. Sem. Univ. Hamburg 70 (1934), 263-284.

3. S. E. Cappell and J. L. Shaneson, Cyclic branched covering spaces Ann. of Math. Studies (Memorial Volume in honor of R. Fox).

4. A. H. Clifford, On the canonical form and dissection of a Riemann's surface, Proc. London Math. Soc. 8 (1877), 292-304.

${ }^{3}$ For $M$ a homotopy 3-sphere, $\mu(M)=0$ if $M \times S^{1} \times S^{1}$ is P.1. homeomorphic to $s^{3} \times s^{1} \times s^{1}$ and $\mu(M) \neq 0$ if $M \times S^{1} \times S^{1}$ is P.1. homeomorphic to the exotic manifold described in [14] which is homotopy equivalent but not P.l. homeomorphic to $s^{3} \times s^{1} \times s^{1}$. 
5. R. H. Fox, A quick trip through knot theory, Topology of 3-Manifolds and Related Topics (Proc. Univ. of Georgia Inst., 1961), Prentice-Hall, Englewood Cliffs, N. J., 1962, pp. 120-167. MR 25 \#3522.

6. Construction of simply connected 3-manifolds, Topology of 3-Manifolds and Related Topics (Proc. Univ. of Georgia Inst., 1961), Prentice-Hall, Englewood Cliffs, N. J., 1962, pp. 213-216. MR 25 \#3539.

7. H. Hilden, Every closed orientable 3-manifold is a 3-fold branched covering space of $S^{3}$, Bull. Amer. Math. Soc. 80 (1974), 1243-1244.

8. F. Hirzebruch, W. P. Newmann and S. S. Koh, Differentiable manifolds and quadratic forms, Dekker, 1971.

9. M. Montesinos, $A$ representation of closed, orientable 3-manifolds as 3-fold branched coverings of $S^{3}$, Bull. Amer. Math. Soc. 80 (1974), 845-846.

10. K. Perko, On dihedral covering spaces of knots (to appear).

11. - Unpublished tables available upon request to the author, 1 Chase Manhattan Plaza, New York, N. Y.

12. K. Reidemeister, Knoten Theorie, Springer-Verlag, 1932.

13. V. A. Rohlin, New results in the theory of four-dimensional manifolds, Dokl. Akad. Nauk SSSR 84 (1952), 221-224. (Russian) MR 14, 573.

14. J. L. Shaneson, Wall's surgery obstruction groups for $G \times Z$, Ann of Math. (2) 90 (1969), 296-334. MR 39 \#7614.

COURANT INSTITUTE OF MATHEMATICAL SCIENCES, NEW YORK UNIVERSITY, NEW YORK, NEW YORK 10012

DEPARTMENT OF MATHEMATICS, RUTGERS UNIVERSITY, NEW BRUNSWICK, NEW JERSEY 08903 